\title{
The large-mass regime of confining but nearly conformal gauge theories
}

\author{
Maarten Golterman* \\ Department of Physics and Astronomy, San Francisco State University, \\ San Francisco, CA 94132, USA \\ and \\ Department of Physics and IFAE-BIST, Universitat Autònoma de Barcelona, \\ E-08193 Bellaterra, Barcelona, Spain \\ E-mail: maarten@sfsu.edu
}

\section{Yigal Shamir}

Raymond and Beverly Sackler School of Physics and Astronomy, Tel Aviv University, 69978, Tel Aviv, Israel

E-mail: shamirepost.tau.ac.il

\begin{abstract}
We apply a recently developed dilaton-pion effective field theory for asymptotically free gauge theories near the conformal window to the $S U(3)$ gauge theory with $N_{f}=8$ fermions in the fundamental representation. Numerical data for this theory suggest the existence of a large-mass regime, where the fermion mass is not small but nevertheless the effective theory is applicable because of the parametric proximity of the conformal window. In this regime, we find that the mass dependence of hadronic quantities is similar to that of a a mass-deformed conformal theory, so that distinguishing infrared conformality from confinement requires the study of subleading effects.
\end{abstract}

The 36th Annual International Symposium on Lattice Field Theory - LATTICE2018

22-28 July, 2018

Michigan State University, East Lansing, Michigan, USA.

${ }^{*}$ Speaker. 


\section{Introduction}

Triggered by numerical results $[1,2,3,4]^{1}$ obtained in simulations of gauge theories with fermions which are generally believed to be near the conformal window, we developed a systematic effective field theory (EFT) to describe the long-distance behavior of such theories, specifically, those with an $S U\left(N_{c}\right)$ gauge group with $N_{f}$ fermions in the fundamental representation [5]. In this talk, we report on more recent work aiming to compare the EFT directly with numerical data [6]. In particular, Ref. [6] made the following claims:

- Even if the fermion mass of a near-conformal theory is large compared to the chiral-symmetry breaking/confinement scale, it can be described systematically by the EFT.

- In this large-mass regime, it is very hard to distinguish such a theory from a mass-perturbed theory whose massless limit is conformal in the infrared.

- In the case of the $S U(3), N_{f}=8$ theory, either much smaller fermion masses or a much higher precision will be needed in current simulations.

The rest of this talk will be concerned with a brief review of the explanations for these claims.

\section{The effective field theory}

Figure 1 shows the hadron spectrum for $S U(3)$ gauge theory with $N_{f}=8$ degenerate fundamental flavors, as a function of the fermion mass $m$. Quite unlike the more familiar case of QCD, the spectrum contains a very light, and, for these fermion masses, stable $0^{++}$state, nearly degenerate with the pseudo-Nambu-Goldstone bosons for the $S U(8)_{L} \times S U(8)_{R}$ chiral symmetry breaking, which we will refer to as the pions.

Clearly, any EFT aiming to describe the low-energy pion physics should also contain a field for the $0^{++}$state, which, in light of what follows, we will refer to as the dilatonic meson (dilaton, for short). In order to construct such a theory, we made the following assumptions [5]:

- Scale invariance gets restored as we take the theory closer to the conformal window. For $N_{f}$ fundamental flavors in an $S U\left(N_{c}\right)$ theory this happens when $N_{f}$ crosses into the conformal region. Technically, $n_{f} \equiv N_{f} / N_{c}$ attains the critical value $n_{f}^{*}$ at this crossing in the limit $N_{c}$, $N_{f} \rightarrow \infty$.

- The theory contains pions associated with chiral symmetry breaking, and a dilaton associated with breaking of scale symmetry, which becomes massless for $n_{f} \rightarrow n_{f}^{*}$ (and $m \rightarrow 0$ ).

- The dilaton potential has a zero, as a function of the dilaton field $\tau$.

Under these assumptions, the following leading-order (LO) lagrangian was constructed in Refs. [5, 6]:

$$
\mathscr{L}=\frac{\hat{f}_{\pi}^{2}}{4} e^{2 \tau} \operatorname{tr}\left(\partial_{\mu} \Sigma^{\dagger} \partial_{\mu} \Sigma\right)+\frac{\hat{f}_{\tau}^{2}}{2} e^{2 \tau}\left(\partial_{\mu} \tau\right)^{2}
$$

\footnotetext{
${ }^{1}$ The three latter references quoted here represent the most recent numerical work. For more extensive earlier references, see Ref. [5].
} 


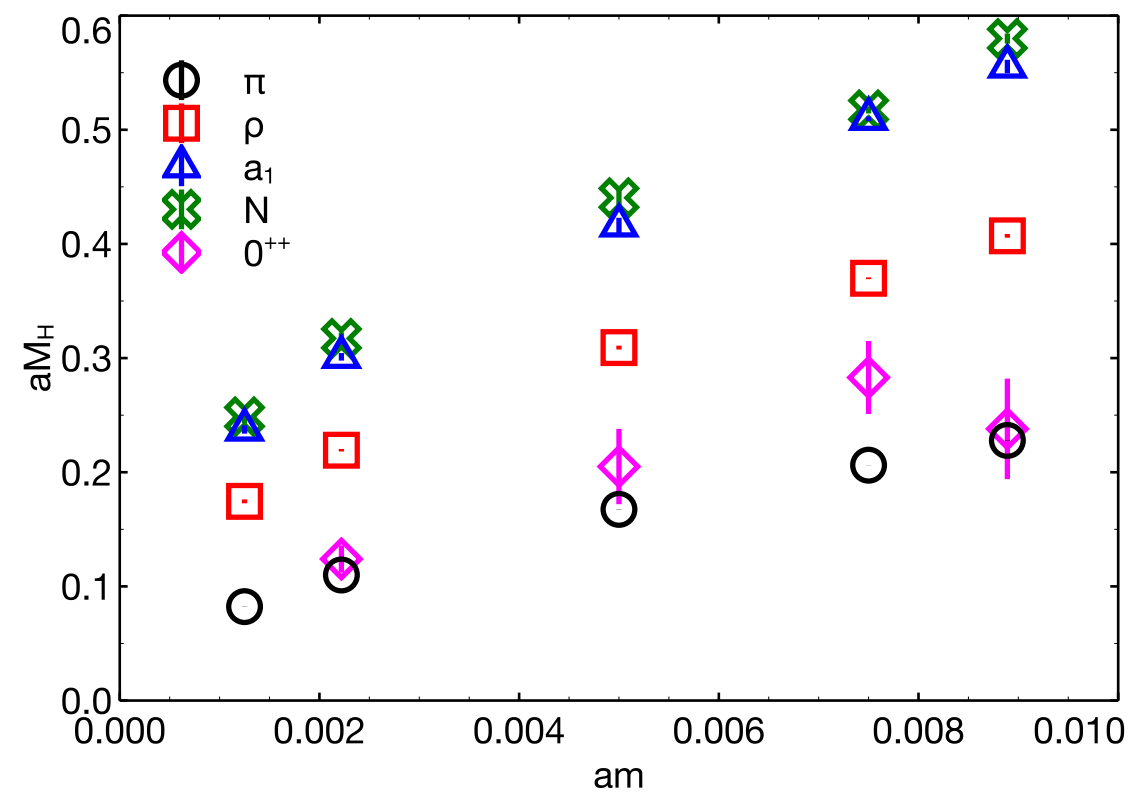

Figure 1: The hadron spectrum for $S U(3)$ gauge theory with $N_{f}=8$ degenerate fundamental flavors, from Ref. [1].

$$
-\frac{\hat{f}_{\pi}^{2} \hat{B}_{\pi} m}{2} e^{\left(3-\gamma_{*}\right) \tau} \operatorname{tr}\left(\Sigma+\Sigma^{\dagger}\right)+\hat{f}_{\tau}^{2} \hat{B}_{\tau} e^{4 \tau} c_{1}\left(\tau-\frac{1}{4}\right)
$$

Here $\gamma_{*}$ is the value of mass anomalous dimension at the infrared fixed point at $n_{f}=n_{f}^{*}$. Along with the mass $m$, the parameter $c_{1} \propto n_{f}-n_{f}^{*}$ is assumed to be small, and the EFT allows a systematic expansion in these small parameters. The $\tau$ field has been shifted so that its LO expectation value $v \equiv\langle\tau\rangle=0$ when $m=0$.

\section{Lowest-order predictions}

At non-vanishing $m$, the $\mathrm{LO}$ value for $v$ satisfies the equation

$$
\frac{m}{c_{1} \hat{M}}=v e^{\left(1+\gamma_{*}\right) v}
$$

where

$$
\hat{M}=\frac{4 \hat{f}_{\tau}^{2} \hat{B}_{\tau}}{\hat{f}_{\pi}^{2} \hat{B}_{\pi} N_{f}\left(3-\gamma_{3}\right)} .
$$

We note that this is an "order-1" equation: both $m$ and $c_{1}$ are small, and we are assuming a power counting in which they are parametrically of the same size. 
If $m$ is large enough that the left-hand side of Eq. (3.1) is much larger than one (we will refer to this range as the "large-mass regime"), we find the approximate solution

$$
e^{v} \approx\left(\frac{m}{c_{1} \hat{M}}\right)^{1 /\left(1+\gamma_{*}\right)} .
$$

From this, it follows that all masses and decay constants scale as $m^{1 /\left(1+\gamma_{*}\right)}$. In other words, even though the theory is in the phase in which chiral symmetry is broken spontaneously at $m=0$, all masses and decay constants exhibit hyperscaling behavior as in a mass-perturbed conformal theory [7]!

In more detail, let us see how this arises for a few examples. Using exact LO results for physical quantities, and then relation (3.3), we find, for instance,

$$
\begin{aligned}
F_{\pi} & =\hat{f}_{\pi} e^{v} \propto \hat{f}_{\pi}\left(\frac{m}{c_{1}}\right)^{1 /\left(1+\gamma_{*}\right)}, \\
M_{\pi}^{2} & =2 \hat{B}_{\pi} m e^{\left(1-\gamma_{*}\right) v} \propto 2 \hat{B}_{\pi} c_{1}\left(\frac{m}{c_{1}}\right)^{2 /\left(1+\gamma_{*}\right)}, \\
M_{\text {nucleon }} & =M_{0} e^{v} \propto M_{0}\left(\frac{m}{c_{1}}\right)^{1 /\left(1+\gamma_{*}\right)}
\end{aligned}
$$

where $M_{0}$ is the nucleon mass in the chiral limit. ${ }^{2}$ All expressions on the right-hand side of these equations show the claimed large-mass hyperscaling behavior.

These results are valid as long as loop corrections are small. As usual, loop corrections are governed by loop expansion parameters such as $M_{\pi}^{2} /\left(4 \pi F_{\pi}\right)^{2}$ and $M_{\tau}^{2} /\left(4 \pi F_{\tau}\right)^{2}$. Considering the first of these two, we find using the exact LO relations in Eq. (3.4) and Eq. (3.1), that

$$
\frac{M_{\pi}^{2}}{\left(4 \pi F_{\pi}\right)^{2}}=\frac{2 c_{1} \hat{M} \hat{B}_{\pi}}{\left(4 \pi \hat{f}_{\pi}\right)^{2}} v
$$

This is small as long as $c_{1} \log m$ is small, where we used that, in the large-mass regime, $v \sim \log m$. This establishes the range of validity of the EFT; for masses larger than this value, loop corrections become too large for the loop expansion defined by the EFT to be useful.

\section{Comparison with numerical results}

From our result, that in the large-mass regime all masses and decay constants exhibit hyperscaling, it follows that ratios of masses and decay constants are approximately constant in this regime. Indeed, results obtained in Ref. [1], shown in Fig. 2, show approximate constancy of the ratio of five different hadron masses with the pion decay constant, as a function of $m$.

Of course, we need to check whether our LO analysis applies to these data. Indeed, the value of the expansion parameter (3.5) is roughly equal to 0.1 for the ratios shown in Fig. 2. However, as also discussed in Ref. [8], it is possible that loop corrections to some quantities are larger than suggested by this value.

\footnotetext{
${ }^{2}$ Note that for small $m$, the solution (3.3) is not valid, and the nucleon mass does not vanish in the chiral limit, as long as $n_{f}<n_{f}^{*}$.
} 


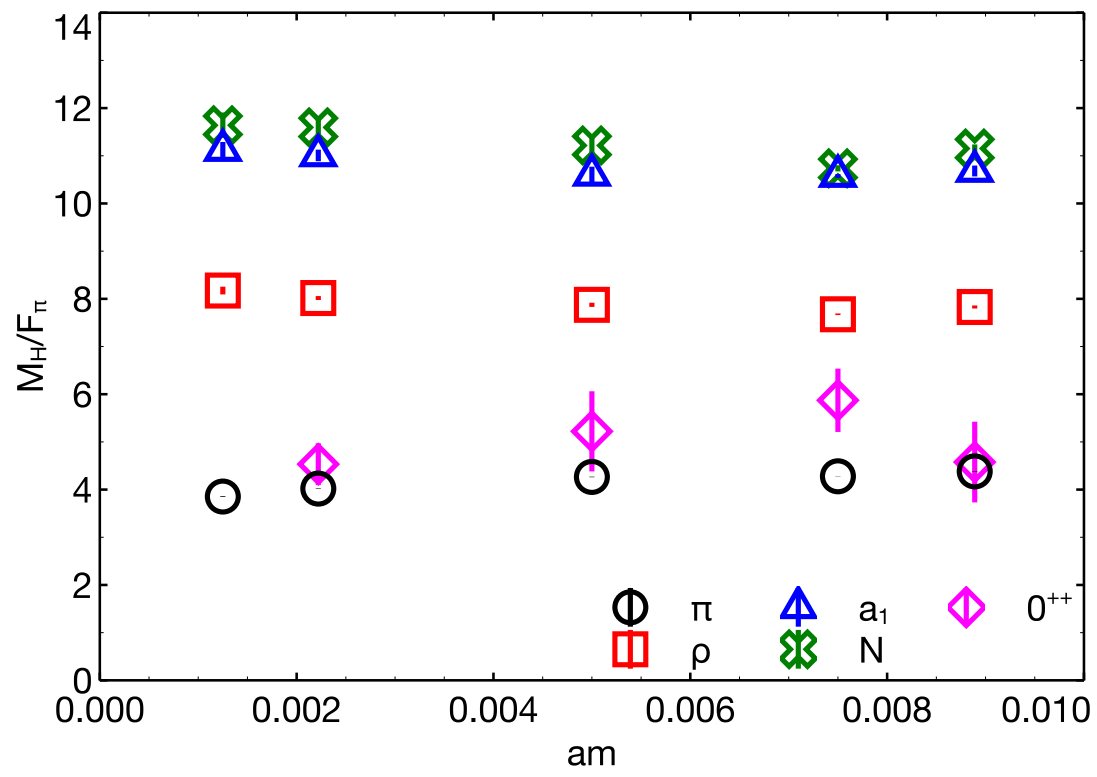

Figure 2: The hadron spectrum for $S U(3)$ gauge theory with $N_{f}=8$ degenerate fundamental flavors, from Ref. [1].

The consistency between our hyperscaling prediction and the data strongly suggests that the values of $m$ investigated in Ref. [4] are in the large-mass regime. And indeed, this turns out to be the case. First, fitting the quantity $M_{\pi}^{2} F_{\pi}^{-1+\gamma_{*}}=2 \hat{B}_{\pi} \hat{f}^{-1+\gamma_{*}} m$ as a function of $m$ yields the estimate $\gamma_{*} \approx 1$ [8]. Using this, and values for the nucleon mass as a function of $m$ obtained in Ref. [4], we find that at the lightest value of $m[6]$,

$$
a m=0.00125 \quad \Rightarrow \quad \frac{m}{c_{1} \hat{M}} \sim 100 .
$$

This implies that, to unambiguously determine whether this theory is conformal or breaks chiralsymmetry in the massless limit, we need either much smaller masses, or enough precision to disentangle subleading effects to the large-mass behavior.

As an additional point of interest, we also find from the numerical data that $\hat{B}_{\pi} / \hat{f}_{\pi} \sim 10^{3}$, which may be a sign of "condensate enhancement" in this theory.

\section{Conclusion}

We briefly summarize our conclusions, referring to Ref. [6] for much more detail.

First, we find that in near-conformal theories, there exists a "large-mass" regime, defined by $m /\left(c_{1} \hat{M}\right) \gg 1$, while the region of applicability of the EFT is, parametrically, $c_{1} \log m \ll 1$. In this regime, masses and decay constants show approximate hyperscaling like in a mass-deformed conformal theory, even though the theory breaks chiral symmetry at $m=0$. 
Second, we find that current simulations in the $S U(3), N_{f}=8$ theory are in the large-mass regime, with $m /\left(c_{1} \hat{M}\right) \geq 100$. Therefore, in this theory, either much smaller masses or much higher precision will be needed to disentangle subleading effects, and distinguish, numerically, between a mass-deformed conformal theory and a theory with spontaneous chiral symmetry breaking in the massless limit.

Summarizing, assuming the validity of our EFT, it provides a possible explanation why it is so difficult to distinguish a conformal theory from a chiral-symmetry breaking theory near the conformal sill.

\section{Acknowledgements}

This material is based upon work supported by the U.S. Department of Energy, Office of Science, Office of High Energy Physics, under Award Number DE-FG03-92ER40711 (MG). YS is supported by the Israel Science Foundation under grant no. 491/17.

\section{References}

[1] T. Appelquist et al., Strongly interacting dynamics and the search for new physics at the LHC, Phys. Rev. D 93, no. 11, 114514 (2016) [arXiv:1601.04027 [hep-lat]].

[2] Y. Aoki et al. [LatKMI Collaboration], Light flavor-singlet scalars and walking signals in $N_{f}=8$ QCD on the lattice, Phys. Rev. D 96, no. 1, 014508 (2017) [arXiv:1610.07011 [hep-lat]].

[3] Z. Fodor, K. Holland, J. Kuti, D. Nogradi and C. H. Wong, The twelve-flavor $\beta$-function and dilaton tests of the sextet scalar, EPJ Web Conf. 175, 08015 (2018) [arXiv:1712.08594 [hep-lat]].

[4] T. Appelquist et al. [Lattice Strong Dynamics Collaboration], Nonperturbative investigations of SU(3) gauge theory with eight dynamical flavors, arXiv:1807.08411 [hep-lat].

[5] M. Golterman and Y. Shamir, Low-energy effective action for pions and a dilatonic meson, Phys. Rev. D 94, no. 5, 054502 (2016) [arXiv:1603.04575 [hep-ph]].

[6] M. Golterman and Y. Shamir, The large-mass regime of the dilaton-pion low-energy effective theory, Phys. Rev. D 98, 056025 (2018) [arXiv:1805.00198 [hep-ph]].

[7] L. Del Debbio, B. Lucini, A. Patella, C. Pica and A. Rago, The infrared dynamics of Minimal Walking Technicolor, Phys. Rev. D 82, 014510 (2010) [arXiv:1004.3206 [hep-lat]]. L. Del Debbio and R. Zwicky, Hyperscaling relations in mass-deformed conformal gauge theories, Phys. Rev. D 82, 014502 (2010) [arXiv:1005.2371 [hep-ph]].

[8] T. Appelquist, J. Ingoldby and M. Piai, Dilaton EFT Framework For Lattice Data, JHEP 1707, 035 (2017) [arXiv:1702.04410 [hep-ph]]; Analysis of a Dilaton EFT for Lattice Data, JHEP 1803, 039 (2018) [arXiv:1711.00067 [hep-ph]]. 